\section{A Brief Introduction of ABC2017}

Zhang Yun ${ }^{1}$,Tong Xiao-fei ${ }^{1}$, Zhou Zi-yu², Yu Hai-yang ${ }^{2}$, Zhang Bin², Liu An-song², Gan Rong-xing\#, Huang Ju-fang*

$1,2,{ }^{*}$ Discipline of Anatomy and Neurobiology, Basic Medical school, Central South University, Changsha 410011, Hunan Province, P.R. China

\# Shanghai Clinical Research Center, 2F, Building 4, No.188, Pingfu Road, Xuhui District, Shanghai 200231, P.R. China

The tradition of the Annual Biobank China (ABC) Conference is as an interactive and innovative conference experience which promotes the development of the Chinese biobanking system and offers a great platform to provide further education to Chinese biobankers. Six ABC conferences have established a global network of professionals, providing the opportunity and momentum for major progress in the development of biobanking and related medical research.

The Annual Biobank China 2017 \& International Symposium on Precision Medicine, ( $A B C 2017)$, is the latest $A B C$ meeting. $A B C 2017$ was successfully held in Changsha, China on December 1st-2nd, 2017, organized by the Central South University (CSU), with invaluable assistance from the Shanghai Clinical Research Center (SCRC) and the Shanghai Engineering Research Center of Biobank (SERCB)

ABC2017 succeeded in inviting more than 100 leading biobanking researchers as speakers. It also attracted more than 1000 participants from 8 countries worldwide who were mainly biobankers, future biobankers, bio-technological professionals, academics, other top scientists, manufacturers, and policy makers. Comprising five preliminary plenary symposia, five preliminary parallel sessions, two seminars and two special interest group programs, ABC2017 reached consensus on quality control and standardization systems, ethical and regulation issues, sustainable development strategy and application of big data, automation and artificial intelligence in biobanking construction and management. ABC2017 also promoted a broad discussion on precision medicine and next-generation biobank construction between biobankers operating under different laws and ethical regulations, different networks, different business models and variable development status in different countries, and different governmental strategies. During this meeting, the Hunan Industry Technology Innovation Strategic Alliance was founded. In addition, a new automated liquid nitrogen storage system was released, the HatchTM from Genepoint Biological Technology (Shanghai) Co. Ltd. In particular, ABC2017 was the first ABC meeting which held a photographic exhibition, showing the history of biobanking in China and interesting stories from biobankers across the world.

We would like to extend our special thanks to Prof. Jim Vaught for his guidance and assistance during May to December 2017 in the preparation phase of this meeting and arranging for a special issue with topics from ABC2017 in the official journal of ISBER: Biopreservation and Biobanking. We also want to express our gratitude to Prof. Chen Xiang, Prof. Gan Rongxing, Prof. Cao Ya, Prof. Huang Jufang and other chairpersons for their devotion. Lastly, we want to thank Prof. Andrew Brooks, Prof. Jan-Eric Litton and other professionals, companies and all the volunteers for their efforts during this meeting, which not only contributed to the success of this inter-disciplinary, multi-field conference among biobankers, but also will promote worldwide cooperation among country-based health and technology programs.

This work was funded by the National Key Research and Development Program of China (2016YFC1201800).

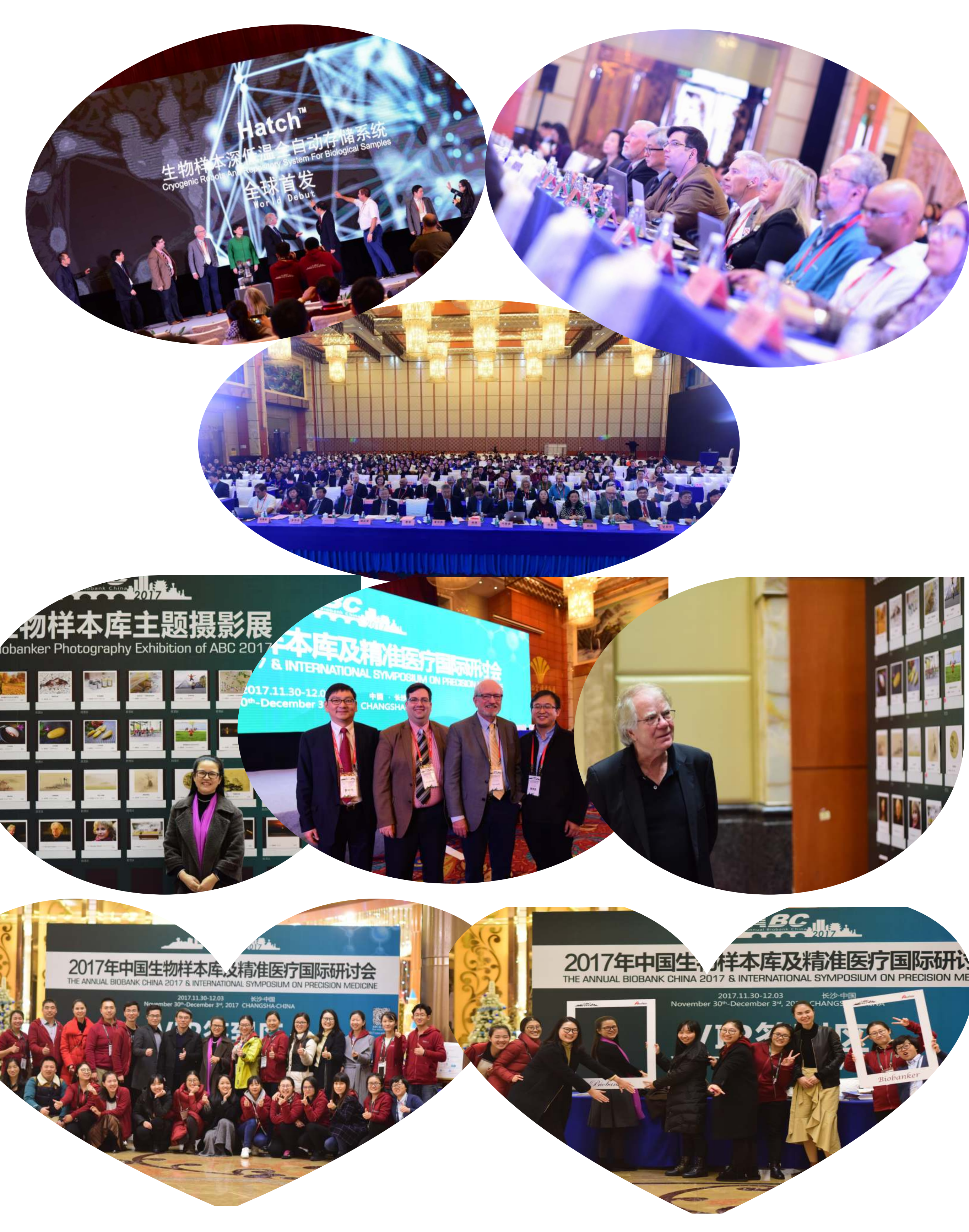

